Historic, Archive Document

Do not assume content reflects current scientific knowledge, policies, or practices. 



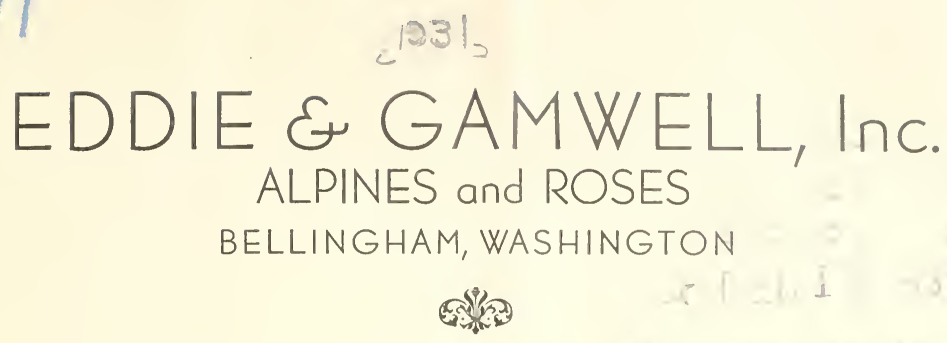

3. Dexarmer

ricul are.

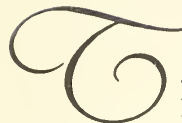
O STIMULATE sales during existing business conditions ye have radically cut prices on our high quality rose bushes. Production costs are less, the percentage of first quality plants is unusually large this season, profits have been slashed and the prices practically reduced to wholesale figures. On all rose bushes except novelties, scarce varieties and those that are hard to propagate, we now make a uniform price of $50 \mathrm{c}$ per bush.

Orders for five or more bushes will be delivered free by mail or express. On orders for one hundred or more, in any one shipment, a further discount will be given. This should interest community clubs, garden societies, park superintendents and other large buyers.

\section{Shrub Roses}

Varieties marked Shrub Roses are those of erect, bushy type adapted to base planting, individual lawn specimens, hedges, or to border a garden of smaller growing roses and thus are valuable features of any planting. They need little pruning and are not at all fussy about soil or cultural conditions.

\section{Polyantha Roses}

The Polyantha Roses are dwarf, bushy plants bearing many clustered flowers in profusion, constantly produced. They are especially valuable for massing in groups and for edging lawns, parking strips, or rose gardens. They are scarcely ever out of flower from June to the Autumn frosts and require no pruning other than to keep the plants shapely.

\section{Climbing Roses}

In the list of Climbing Roses are found those of the popular rambler or Wichuriana type, useful for any condition where rapid flexible growth is desired, and there are also listed the climbing forms of the hybrid teas and Pernetianas. These are valuable for pillar roses, or to be supported by stakes and give the same type of bloom produced by their dwarf namesakes.

\section{Novelties} herein.

At somewhat advanced prices we can supply most of the novelties not listed

The low prices quoted are temporary only, due to Depression Times, and do not indicate inferior quality, nor any decrease of our usual high standard stock.

All our roses are grown two years in the open field and are guaranteed to be budded onto appropriate understocks which give them the strength and stamina so ardently desired by all rose lovers.

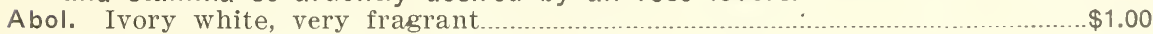

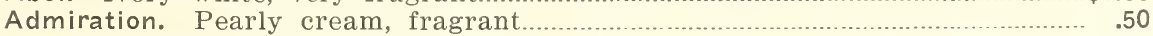

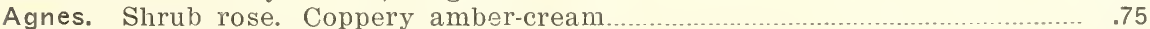

Alice Amos. Poly. Cherry pink, white eye

Amelie de Bethune. Coral red

Angele Pernet. Orange and gold

Antoine Rivoire. Golden flesh

Ariel. Orange flame, shaded crimson $\ldots .75$

Arthur R. Goodwin. Coppery orange red.

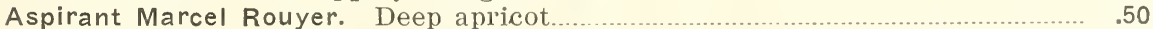

Austrian Copper. Intense coppery-red.

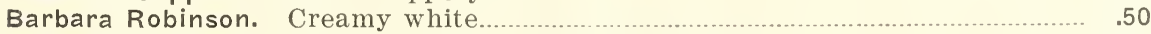

Betty. Pink buds of exquisite form, opening copper rose.................................. $\quad .50$

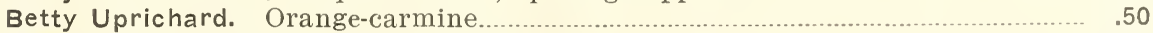

Bloomfield Abundance. Salmon-pink

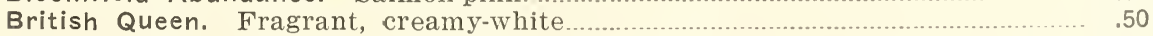

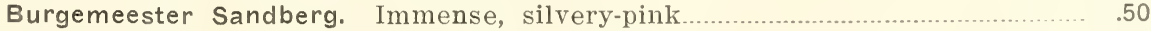

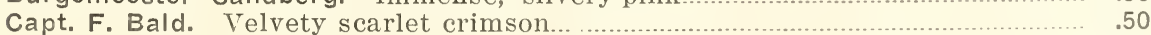




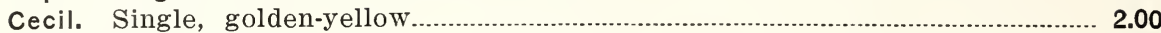

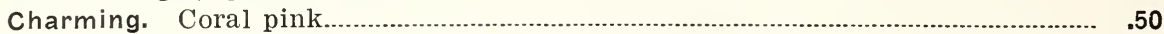

Charles K. Douglas. Fragrant, flaming scarlet..................................................... .50

Chateau de Clos Vougeot. Dark velvety garnet................................................... .50

Cheerful. Shining pink illumined orange................................................................ .50

Chrissie Mackellar. Delicate orange-pink............................................................. .50

Christine. Deep golden-yellow ................................................................................... .50

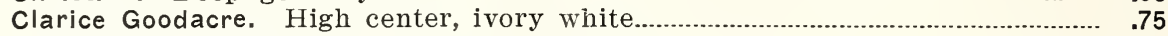

Col. Oswald Fitzgerald. Dark velvety-crimson......................................................... .50

Columbia. Pointed bud, glistening pink............................................................... 50

Comte G. de Rochemur. Fiery scarlet..............................................................

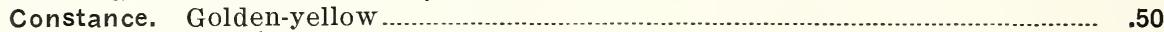

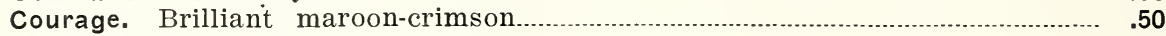

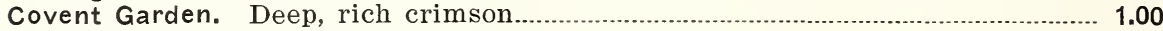

Crimson Emblem. Dazzling crimson...................................................................... 1.00

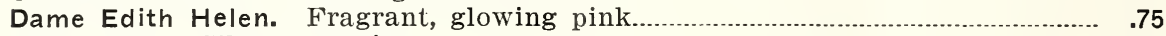

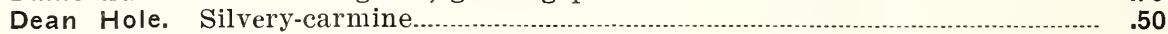

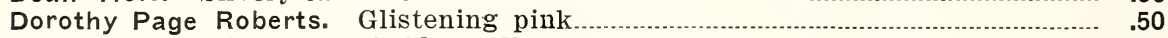

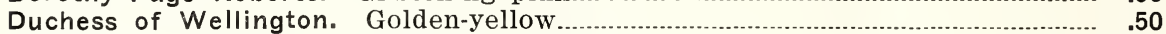

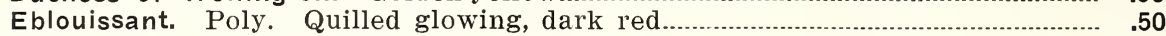

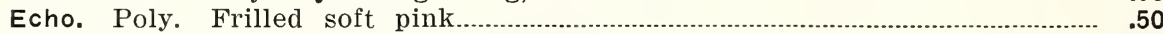

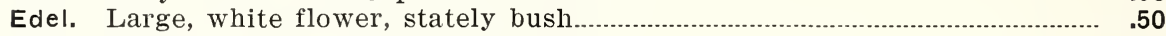

Edith Cavell. High centered, lemon-white........................................................... $\quad .50$

Ellen Poulsen. Poly. Bright rose-pink................................................................. .50

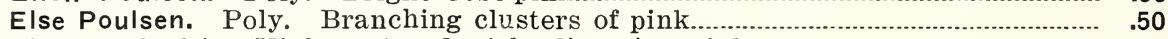

Elsie Beckwith. High centered, rich glistening pink............................................ .75

Elvira Aramayo. Slender, scarlet buds, reddish-copper flowers................................50

Edward Mawley. Fragrant, dark crimson............................................................ $\quad .50$

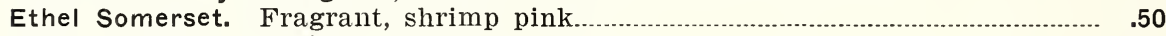

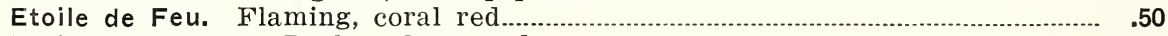

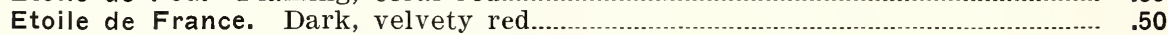

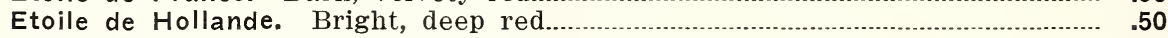

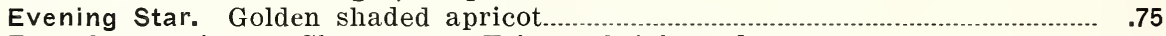

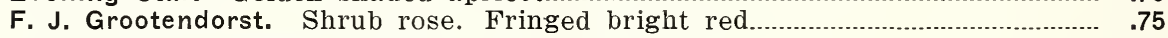

Feu Joseph Looymans. Lasting Indian yellow ....................................................

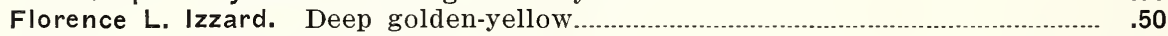

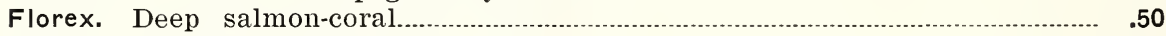

Francis Scott Key. Deep Tyrian pink

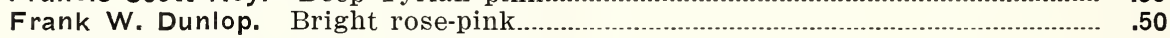

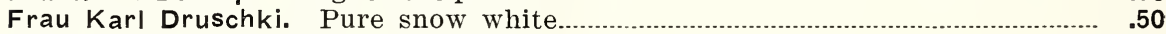

Geheimrat Dr. Mittweg. Rosy pink

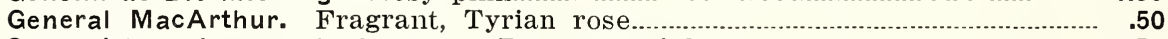

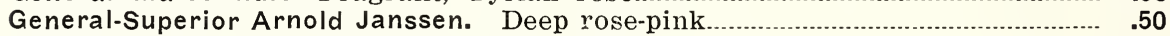

Georg Ahrends. Deliciously fragrant, pink

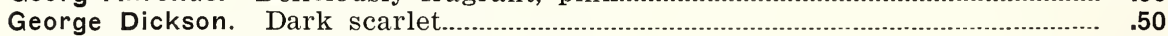

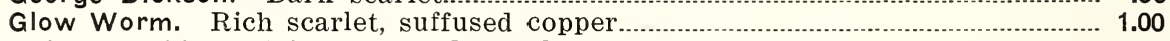

Golden Emblem. Vigorous golden-yellow............................................................

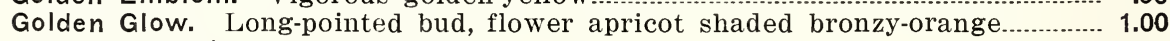

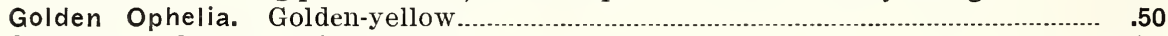

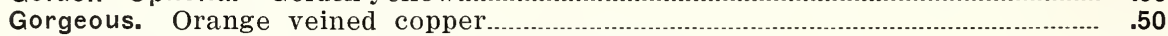

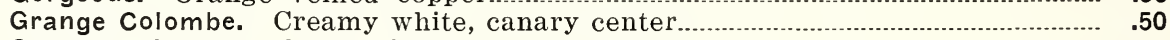

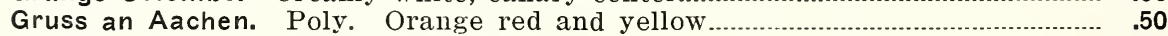

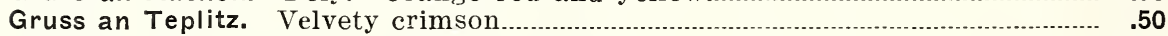

Gwyneth Jones. Brilliant carmine-pink

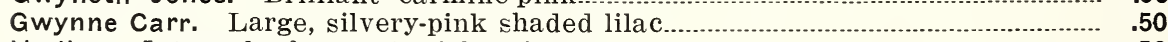

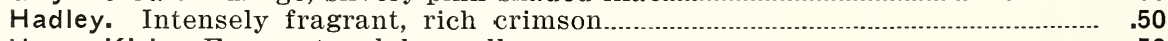

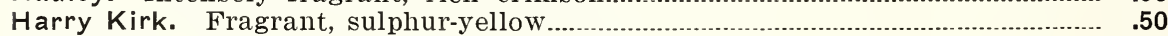

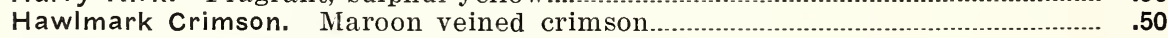

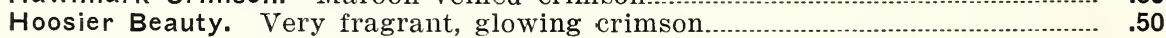

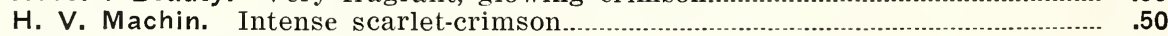

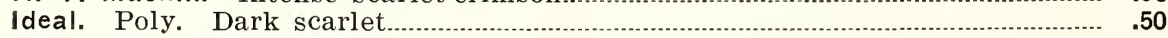

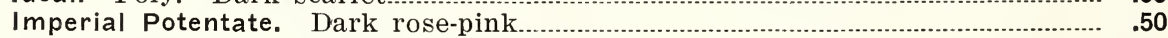

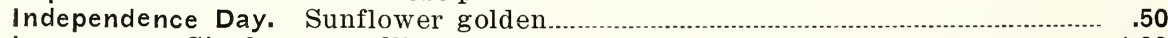

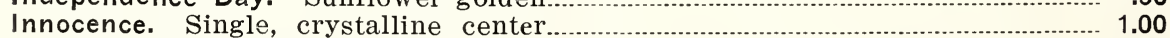

Isobel. Single, light rose-pink

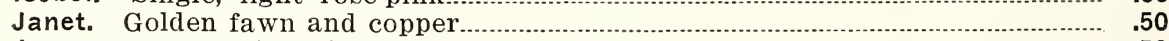

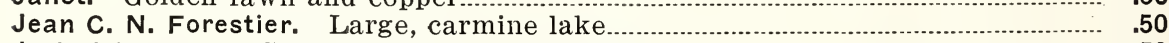

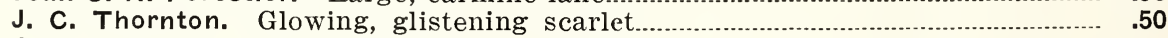

J. G. Glassford. Clear, deep crimson lake 
Joanna Hill. Pointed bud, rich yellow flower.......... 1.50

Jonkherr J. L. Mock. Silvery rose, reverse carmine...........

John Russell. Rich, glowing crimson........................................

J. Otto Thilow. Rich rose, reflexed petals................................

Joyous Cavalier. Vigorous, brilliant scarlet.......................................

Juliet. Glowing pink, rich old gold on reverse................................

Kaiserin Auguste Viktoria. Snowy white, center lemon............ 50

Kardinal Piffl. Fawn red inside, golden reverse.......................

Killarney Brilliant. Perfumed, brilliant pink .......................... 50

Killarney Queen. Semi-single, Tyrian rose............................. $\quad .50$

K. of K. Intensely fragrant, pure, dazzling scarlet..................

Konigin Corola. Satiny, rose-pink......................................

Lady Ashtown. Carmine pink, yellow base.........................

Lady Alice Stanley. Pale flesh-pink..........................................

Lady Craig. Deep yellow ......................................................

Lady Florence Stronge. Reddish prawn....................................

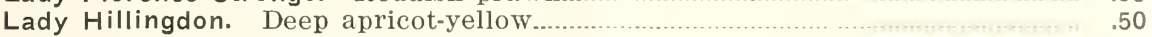

Lady Inchiquin. Orange vermillion.......................................

Lady Margaret Stewart. Buttercup yellow

Lady Pirrie. Coppery reddish salmon.................................

Lady Roundway. Deep coppery-orange.........................................

Lady Ursula. Clear cameo pink .............................................

Li Bures. Gold Medal. Blended rose red and yellow................... 1.00

Lord Charlemont. Clear, deep crimson ................................................

Lord Lambourne. Deep buttercup, edged carmine........................................

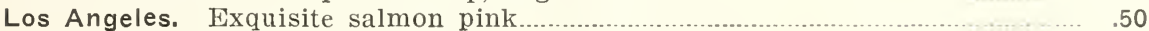

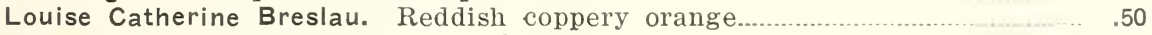

Mabel Morse. Fragrant, bright yellow ...........................................................

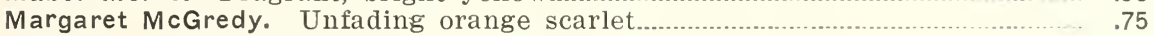

Mari Dot. Gold Medal. Reddish salmon, yellow base...................................... 1.50

Marie Van Houtte. Deep crimson, tinged carmine.......................................... .50

May Wettern. Gold Medal. Fragrant, rosy-pink............................................ 1.00

Mevrouw G. A. Van Rossem. Highly colored golden-yellow, reverse deep

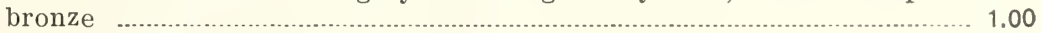

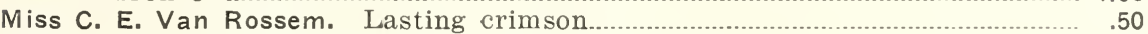

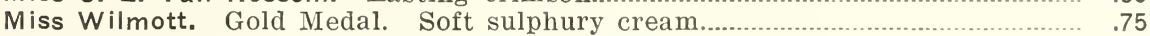

Mme. Butterfly. Exquisite shape. Pink tinted gold …...................................... 50

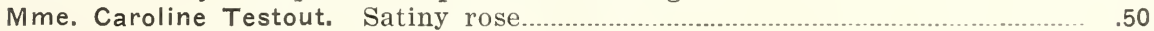

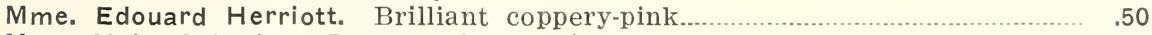

Mme. Meha Sabatier. Large, velvety crimson....................................................... 50

Moonlight. Shrub rose. Gold medal. Clustered white, tinted lemon................. .50

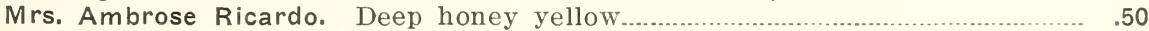

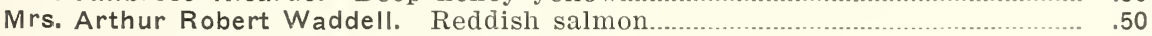

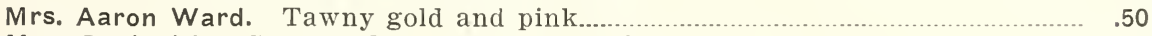

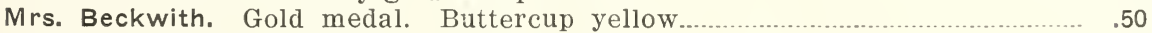

Mrs. Charles Bell. Shell pink shaded salmon............................................ .50

Mrs. Charles E. Russell. Pink American Beauty ............................................5

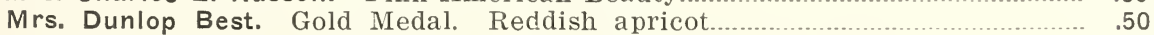

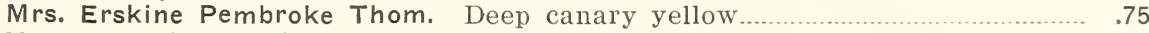

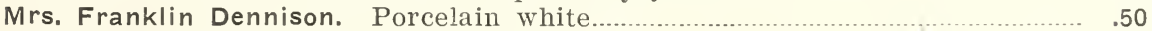

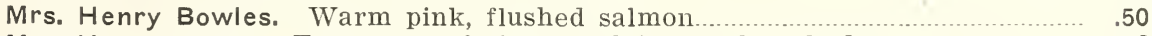

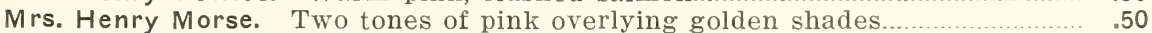

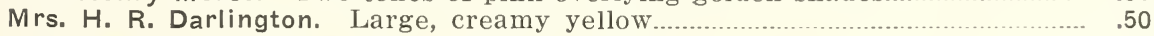

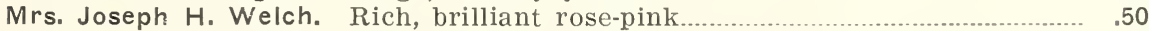

Mrs. Pierre S. du Pont. Bagatelle Gold Medal 1929. Bud long-pointed, reddishgolden, open flower of deep golden-yellow. Disease resistant foliage.... 2.00

Mrs. Redford. Gold Medal. Gracefully reflexed petals of bright apricot........... 1.00

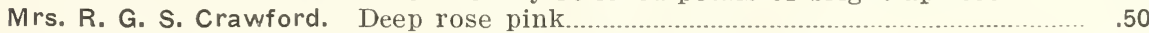

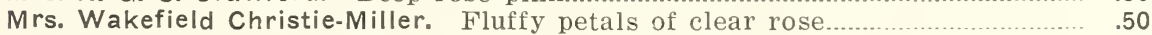

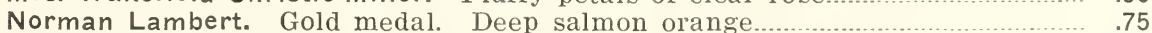

Nuntius Pacelli. Gold medal. Pure white, cream center.................................. 1.00

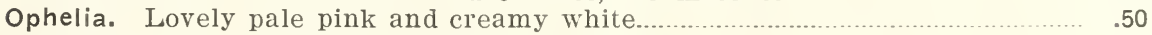

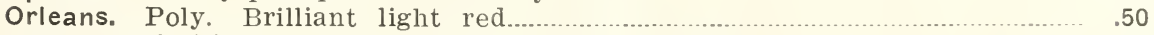

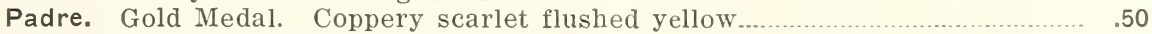

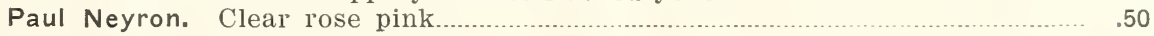

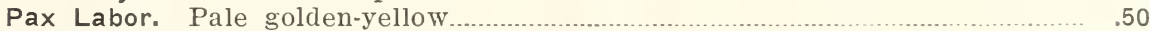

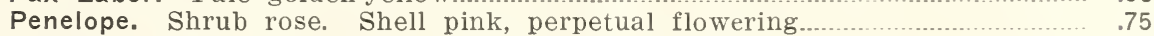

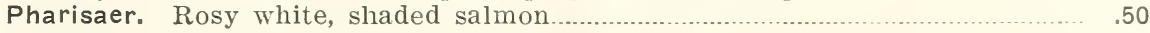

Pink Pearl. Fragrant, deep rose pink

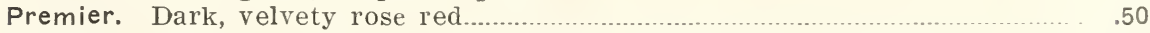

President Cherioux. Bagatelle Gold Medal. Prawn red and salmon................ 1.00 
President Wilson. Large flower, delightful shade of shrimp pink.

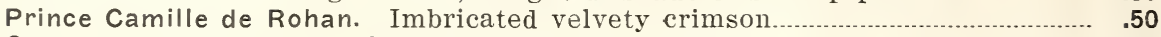

Queen of Fragrance. Shell pink, tipped with silver.

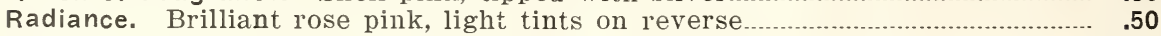

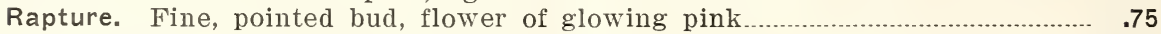

Red Radiance. Free blooming, light crimson................................................

Rev. F. Page Roberts. Coppery-red buds, golden bloom stained red................. $\quad .50$

Rosa xanthina, Allard. Shrub rose. Fine double yellow bloom, fernlike

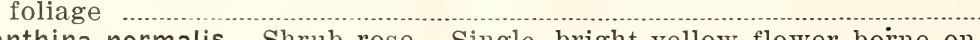

Rosa xanthina normalis. Shrub rose. Single, bright yellow flower borne on

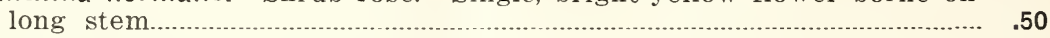

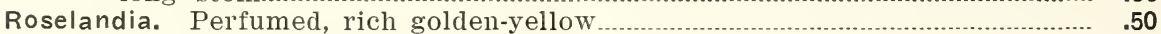

Rose Marie. Fragrant, rose pink

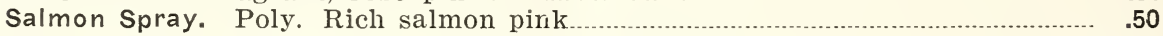

Sammy. Shrub rose. Clusters of bright carmine $\ldots$

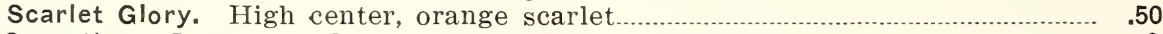

Sensation. Large, scarlet crimson flower ….................................................... 50

Shot silk. Cherry cerise shot with golden shades......................................... $\quad .50$

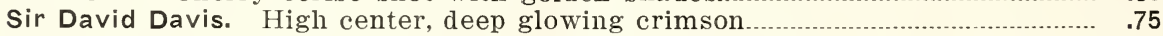

Souv. de Claudius Pernet. Fine buds of fadeless yellow....................................50

Souv. de Georges Pernet. Brick red bud, orange-pink flower....................................50

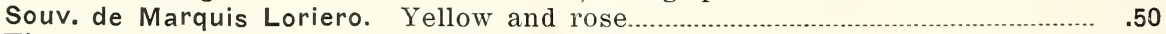

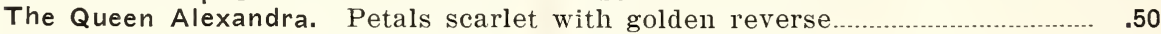

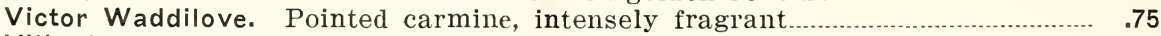

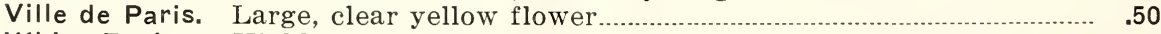

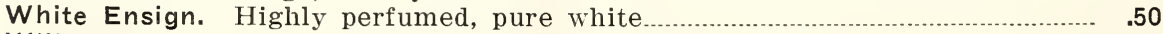

Willowmere. Shrimp pink shaded gold

Wilhelm Kordes. Intensely fragrant, golden yellow coppery edges......................5

\section{Climbing Roses at 75c Each}

Albertine, vermillion-copper

American Beauty, rosy-carmine

American Pillar, bright pink, white center

Aviateur Bleriot, saffron and crimson

Bonnie Prince, frilled white

Breeze Hill, apricot and gold

Christine Wright, wild rose-pink

Dr. Huey, deep crimson-maroon

Dr. Van Fleet, shell pink

Emily Gray, golden yellow

Frau Karl Druschki, snow white

Glenn Dale, pale yellow, double

Gloire de Dijon, buff pink

Golden Emblem, golden-yellow, lasting

Gruss an Teplitz, velvety-crimson
Jacotte, orange-yellow

Los Angeles, exquisite calmon-pink Marechal Niel, golden-yellow

Mary Wallace, double, rosy-pink

Max Graf, prostrate, shining pink

Mme. Butterfly, pink tinted gold

Mme. Caroline Testout, satiny-rose

Mme. Edouard Herriot, coppery-pink

Mrs. Aaron Ward, tawny gold and pink

Paul's Scarlet, intense scarlet

Paul's Lemon Pillar, pale lemon

Rose Marie, fragrant rose-pink

Souv. de Claudius Denoyel, large dazzling red

Star of Persia, single, golden-yellow

\section{Climbing Roses at \$1.00 Each}

Daphne, soft pink flushed rose

Daybreak, single golden-yellow

Chamisso, rosy-flesh

Mme. Gregoire Staechelin, frilled, fragrant, delicate pink
Sammy, clusters of single, bright carmine

Uhland (half climbing) fringed, reddish-yellow clusters

\section{Alpines and Rock Plants}

In addition to roses we offer a large collection of alpines and other rockery plants, many of which are new, unusual and rare types. Strange campanulas, unusual gentians and saxifrages, encrusted and kabschias, as well as mossy types, are offered. Dainty little mountain plants from the European Alps and the high ranges of India and Tibet and the best natives from the mountains of Oregon, Washington and British Columbia are in our collection.

We offer a good selection of a dozen plants, our choice, for $\$ 3.00$ postpaid, and invite the opportunity to quote on any variety and any quantities.

\section{EDDIE \& GAMWELL, Inc. ALPINES and ROSES}

BELLINGHAM -:- -:- WASHINGTON 